Acta Neurochirurgica 26, 179-190 (1972)

(C) by Springer-Verlag 1972

II. Medizinische Klinik (Direktor: Prof. Dr. E. Buchborn) und Neurochirurgische Klinik (Direktor: Prof. Dr. F. Marguth)

der Universität München

\title{
Zur Diagnostik des Diabetes insipidus unter besonderer Berücksichtigung hypophysektomierter Patienten
}

\author{
Von \\ 0. A. Müller, S. Ascher, R. Fahlbusch, F. Hirsch, F. Kluge, H. C. Moll \\ und P. C. Seriba * \\ Mit 1 Abbildung
}

\section{Zusammenfassung}

Verschiedene, am gleichen Patienten durchgeführte Stimulationsteste für die ADH-Sekretion (17-Std.-Durstversuch, Carter-Robbins-Test, osmotische Diurese) zeigen eine ausgezeichnete Korrelation. Auf Grund dieser Ergebnisse werden Richtlinien zur möglichst einfachen Diagnostik einer Konzentrationsstörung vorgeschlagen. In den meisten Fällen kann allein schon auf Grund eines exakt durchgeführten Durstversuchs eine Konzentrationsstörung nachgewiesen (höchste Urinosmolalität $<500 \mathrm{mOsm} / \mathrm{kg}$ ) bzw. ausgeschlossen (höchste Urinosmolalität $>750 \mathrm{mOsm} / \mathrm{kg}$ ) werden. Lediglich bei einer höchsten Urinosmolalität im 17-Std.-Durstversuch zwischen 500 und $750 \mathrm{mOsm} / \mathrm{kg}$ müssen aufwendigere und den Patienten mehr belastende Tests (z. B. Carter-Robbins-Test) eingesetzt worden, um das Vorliegen bzw. den Grad einer Konzentrationsstörung endgültig diagnostizieren zu können. ADH-Injektionen dienen dabei zur Differenzierung zwischen einem ADH-Mangel und einer mangelhaften ADH-Ansprechbarkeit der Niere. Unsere Untersuchungen an hypophysektomierten Patienten $(\mathrm{N}=29)$ zeigen auch bei Patienten ohne polyurisch-polydiptisches Syndrom $(\mathrm{N}=22)$ eine gegenüber Normalpersonen signifikant eingeschränkte Konzentrationsleistung im 17-Std.-Durstversuch.

* Mit Unterstützung der Deutschen Forschungsgemeinschaft (SFB 51). 


\section{Summary}

Concerning the Diagnosis of Diabetes insipidus with Especial Reference to the Hypophysectomized Patient

The results of three different stimulation tests for ADH (17 hours thirst period, Carter-Robbins-test, osmotic diuresis) correlate well in the same patient. Because of this we propose a simplified procedure for the detection of failure to produce a concentrated urine. In most cases merely on the basis of a precisely executed thirst period test, one can either recognize an abnormality of concentration (highest urine osmolality less than $500 \mathrm{mOsm} / \mathrm{kg}$ ), or exlude it (highest urine osmolality greater than $750 \mathrm{mOsm} / \mathrm{kg}$ ). Only when the highest urine osmolality lies between 500 and $750 \mathrm{mOsm} / \mathrm{kg}$ after a 17 hour thirst must more painstaking tests (e.g. Carter-Robbins test) be employed to determine both the presence of and the degree of a failure to produce a concentrated urine. An injection of ADH can be used to differentiate between a deficiency of $\mathrm{ADH}$ and a defective response of the kidneys to ADH. Our investigations on hypophysectomized patients $(n=29)$ showed that also in patients without the syndrome of polyuria-polydypsia $(n=22)$, there was a significantly reduced capacity to concentrate urine during a 17 hour thirst period, as compared with normal people.

\section{Einleitung}

Boi Patienten mit hypothalamischen oder HVL-Tumoren stellt sich im Rahmen der endokrinologischen Diagnostik auch die Aufgabe, Störungen der ADH-Sekretion exakt zu diagnostizieren. Da eine routinemäBig durchführbare Bestimmung des ADH-Gehaltes im Blut bzw. im Serum noch nicht zur Verfügung steht, muß die ADH-Sekretion weiterhin durch indirekte Methoden (Stimulationsteste mit Messung der ADH.Wirkung) überprüft werden.

Mit dieser Arbeit soll der diagnostische Wert des den Patienten relativ wenig beeinträchtigenden Durstversuches geprüft und mit belastenderen Verfahren (Cartcr-Robbins-Test, osmotische Diurese) verglichen werden. Ferner interessierte, inwieweit auch hypophysektomierte Patienten, bei denen klinisch kein ADH-Mangel diagnostiziert wurde, d. h. kein polyurisch-polydiptisches Syndrom vorlag, eine eingeschränkte Konzentrationsleistung aufwiesen.

\section{Patienten}

Wir teilten die untersuchten Patienten in drei Gruppen ein:

A. Patienten nach Hypophyselstomie* ohne ein polyurisch-polydiptisches Syndrom.

B. Patienten nach Hypophysektomie* mit einem polyurisch-polydiptischen Syndrom.

* "Hypophysektomie" ist in diesem Zusammenhang ein pauschaler Begriff für Operationen bei u. a. Hypophysenadenomen und Kraniopharyngiomen. 
C. Patienten mit polyurisch-polydiptischem Syndrom ohne vorausgegangene Hypophysektomie.

Bei allen Patienten wurde ein Durstversuch und zusätzlich - soweit zeitlich möglich und dem Patienten zumutbar — entweder ein CarterRobbins-Test oder eine osmotische Diurese durchgeführt. Kriterium für das Vorliegen eines polyurisch-polydiptischen Syndroms war eine tägliche Ausscheidungsmenge von mehr als zwei Litern. Wegen der Verminderung der Polyurie (Diabetes insipidus) bei gleichzeitiger HVL-Insuffizienz ${ }^{1}$ waren alle Patienten zum Zeitpunkt der Untersuchungen ausreichend mit Cortisol und Schilddrüsenhormonpräparaten substituiert, soweit die endokrinologische Funktionsdiagnostik einen Mangel an diesen Hormonen ergeben hatte. STH wurde auch bei gesicherter Minderung bzw. Ausfall dieses Hormons nicht substituiert. ADH-Präparate bzw. Präparate mit ADHpotenzierender oder die ADH-Sekretion stimulierender Wirkung waren rechtzeitig abgesetzt worden.

\section{Methoden}

Für sämtliche Teste wurde die kryoskopische Osmolalitätsbestimmung in Urin und Serum mittels eines Osmometers der Fa. Knauer, Berlin, eingesetzt. Dieses Verfahren zeichnet sich durch große Einfachheit und Reproduzierbarkeit (Qualitätskontrolle von Tag zu Tag mit einem gepoolten Kontrollserum : $\overline{\mathrm{x}}=292 \mathrm{mOsm} / \mathrm{kg}, \mathrm{s}=3,8 \mathrm{mOsm} / \mathrm{kg}, \mathrm{VK}=1,3 \%, \mathrm{~N}=44$ ) aus und ist damit auch weit exakter als die Messung des spezifischen Gewichtes $^{2-4}$.

Durstversuch: Es wurde - soweit das dem Patienten zuzumuten war eine Flüssigkeitskarenz von 17 Std. eingehalten, der Urin während dieses Zeitraumes in drei Fraktionen gesammelt $(19.00$ bis $8.00 \mathrm{Uhr}, 8.00$ bis $10.00 \mathrm{Uhr}, 10.00$ bis $12.00 \mathrm{Uhr})$ und Blut zur Bestimmung der Serumosmolalität um 19.00, 9.00 und um 11.00 Uhr entnommen. Eine Gewichtskontrolle zu Beginn und nach Beendigung der Durstperiode diente zur Überprüfung der ,Mitarbeit" der Patienten; die Gewichtsabnahme mußte bei exakt durchgeführtem Durstversuch der ausgeschiedenen Flüssigkeitsmenge entsprechen. Das wichtigste Kriterium des Durstversuches war die höchste erreichte Urinosmolalität ${ }^{2},{ }^{3}$. Das Harnvolumen war nur ein grober Anhaltspunkt, da die Flüssigkeitszufuhr vor Beginn des Durstversuches beliebig war. Bei pathologisch ausgefallenen Versuchen konnte die Serumosmolalität zusätzlich als Kriterium für die richtige Durchführung herangezogen werden, da bei ADH-Mangel bzw. mangelhafter ADH-Ansprechbarkeit die Serumosmolalität durch den übermäßigen Wasserverlust anstieg.

Von uns untersuchte endokrinologisch gesunde Probanden zeigten in dem unter diesen Bedingungen durchgeführten Durstversuch eine höchste Urinosmolalität von $973 \pm 103 \mathrm{mOsm} / \mathrm{kg}(\overline{\mathrm{x}} \pm \mathrm{s}, \mathrm{N}=8)$ bei einer ausgeschiedenen Urinmenge von $580 \pm 350 \mathrm{ml} / 17 \mathrm{Std} .(\overline{\mathrm{x}} \pm \mathrm{s})$ und einer über die Dauer des Durstversuches im Mittel konstant bleibenden Serumosmolalität ${ }^{2}$.

Carter-Robbins-Test: Die Versuchsperson. wurde zunächst mit einem Wasserstoß (Tee) in einer Dosierung von $20 \mathrm{ml}$ pro kg Körpergewicht oral belastet und die Urinausscheidung in 15-Minuten-Sammelperioden bestimmt. Bei einem Harnfluß von mehr als $5 \mathrm{ml} / \mathrm{min}$ wurden von einer hypertonen (2,5\%ige) NaCl-Lösung $0,25 \mathrm{ml}$ pro kg Körpergewicht pro min über $45 \mathrm{Mi}$ nuten i. v. infundiert. Nach zwei weiteren 15-Minuten-Sammelperioden, in denen normalerweise der Urinfluß um mehr als $70 \%$ abnimmt und als sichereres Kriterium - die Urinosmolalität um zumindest $300 \mathrm{mOsm} / \mathrm{kg}^{6}$ 
ansteigt, wurden $300 \mathrm{mE}$ Pitressin akut i. v. und weitere $300 \mathrm{mE}$ in $250 \mathrm{ml}$ einer $5 \%$ igen Laevulose-Lösung über $30-45 \mathrm{~min}$ intravenös verabreicht. Auch beim Gesunden kann die Urinosmolalität nach der ADH-Gabe noch einmal um höchstens $100 \mathrm{mOsm} / \mathrm{kg}$ ansteigen ?

Das Ansprechen auf die ADH-Gabe kann bei ausgebliebenem oder zu geringem Anstieg der Urinosmolalität auf die Infusion der hypertonen Kochsalzlösung als differentialdiagnostisches Kriterium für die zentrale gegenüber der renalen Genese eines polyurisch-polydiptischen Syndroms herangezogen werden.

Osmotische Diurese: Ein Maß für die Konzentrationsfähigkeit der Niere ist die tubuläre Rückdiffusionskapazität des Wassers im distalen Tubulus ( $\mathrm{T}_{\mathrm{H}_{2} \mathrm{O}}^{\mathrm{c}}$ ) und der Regressionskoeffizient (b) für die Beziehung osmolale Clearance $\left(\mathrm{C}_{\mathrm{osm}}\right)$ und Urinminutenvolumen $\left(\mathrm{V}_{\mathrm{min}}\right)$ unter einer osmotischen Diurese (Einzelheiten ${ }^{2,3}$ ). Die osmotische Diurese wurde nach einer Flüssigkeitskarenz von $12 \mathrm{Std}$. mit einer 10\%igen Mannit-Lösung (1500 bis $2000 \mathrm{ml}$ mit einer Laufgeschwindigkeit von 12 bis $17 \mathrm{ml}$ pro min) ausgelöst, unter Kontrolle der Inulin- und PAH-Clearance 2 . Nach Erreichen eines Harnflusses von 5 bis $6 \mathrm{ml} / \mathrm{min}$ wurde der Urin in 5 bis 6 Perioden von je $10 \mathrm{~min}$ gesammelt (Blutentnahme alle $10 \mathrm{~min}$ ). Aus Urinvolumen, Urin- und Serumosmolalität wurde für das jeweilige Urinminutenvolumen $\left(V_{\min }\right)$ die osmolale Clearance $\left(\mathrm{C}_{\mathrm{osm}}\right)$ aus der Beziehung $\mathrm{C}_{\mathrm{osm}}=\frac{\mathbf{U}_{\mathrm{osm}}}{\mathbf{S}_{\mathrm{osm}}} \times \mathrm{V}_{\min }$ errechnet und der Regressionskoeffizient $b$ für die Beziehung von $\mathrm{C}_{\text {osm }}$ gegen $\mathrm{V}_{\min }$ mit Hilfe eines programmierten Rechenvorganges (Tischcomputer P 102 der Fa. Olivetti) ermittelt. $\mathbf{T}_{\mathrm{H}_{2} \mathrm{O}}^{\mathrm{c}}$ wurde entweder aus der Beziehung $\mathbf{T}_{\mathrm{H}_{2} \mathrm{O}}^{\mathrm{c}}=\mathrm{C}_{\mathrm{osm}}$ $V_{\text {min }}$ errechnet oder in einem Koordinatensystem mit der Abszisse $V_{\text {min }}$ und der Ordinate $\mathrm{C}_{\mathrm{osm}}$ abgelesen. $\mathrm{T}_{\mathrm{H}_{2} \mathrm{O}}^{\mathrm{c}}$ ergibt sich dabei aus dem waagerechten Abstand der Geraden mit dem Regressionskoeffizienten $\mathrm{b}$ - abgelesen in $\mathrm{ml} / \mathrm{min}$ an der Abszisse - zum sog. isoosmotischen Parameter, das ist die Gerade mit $b=1$. Beim Gesunden erreicht $\mathrm{T}_{\mathrm{H}_{2} \mathrm{O}}^{\mathrm{c}}$ ab einem bestimmten Grenzwert der Diurese einen konstanten, maximalen Betrag (maximale tubuläre Rückdiffusionskapazität $\operatorname{Tm}_{\mathrm{H}_{2} \mathrm{O}}^{\mathrm{c}}$ ). Da beim ADH-Mangel bzw. mangelhafter ADH-Ansprechbarkeit der Niere mit steigender Diurese dagegen immer größere Mengen eines hypotonen Urins ausgeschieden werden (,hypotoner Diuresezuwachs"), d. h. $\mathrm{T}_{\mathrm{H}_{2} \mathrm{O}}^{\mathrm{c}}$ mit zunehmender Diurese immer kleiner und schließlich evt. sogar negativ wird, muß $\mathbf{T}_{\mathrm{H}_{2} \mathrm{O}}^{\mathrm{c}}$ für einen bestimmten, willkürlich festzusetzenden Harnfluß abgelesen werden. Wir ermittelten $\mathrm{T}_{\mathrm{H}_{2} \mathrm{O}}^{\mathrm{O}}$ durch Intra- oder Extrapolation für einen Harnfluß von $20 \mathrm{ml} / \mathrm{min}$ entsprechend den Angaben von ZAK und Mitarb. ${ }^{8}$. Dabei ist zu beachten, daß ein solchermaßen extrapolierter $\mathrm{T}_{\mathrm{H}_{2} \mathrm{O}^{-}}^{\mathrm{c}}$ Wert nichts über die maximale konzentrative Kapazität aussagt, sofern nicht der höchste gemessene $\mathrm{T}_{\mathrm{H}_{2} \mathrm{O}}^{\mathrm{c}}$-Wert bei einem $\mathrm{V}>6 \mathrm{ml} / \mathrm{min}$ bestimmt wurde. Liegt die für die jeweilige Versuchsperson ermittelte Gerade bei den festgelegten $V_{\text {min }}$ oberhalb des isoosmotischen Parameters, so ist $T_{\mathbf{H}_{2} \mathrm{O}}^{\mathrm{c}}$ positiv, anderenfalls also negativ. Schließlich wird der so für $\mathrm{T}_{\mathrm{H}_{2} \mathrm{O}}^{\mathrm{e}}$ ermittelte Wert noch auf $1,73 \mathrm{~m}^{2}$ Körperoberfläche umgerechnet. Ferner übernahmen wir die in der Literatur ${ }^{8}$ angegebenen Normalwerte für $\mathrm{b}=1,033 \pm 0,15(\mathrm{x} \pm 2 \mathrm{~s})$ 
und $\mathbf{T}_{\mathbf{H}_{2} \mathrm{O}}^{\mathrm{c}}=5,1 \pm 3,0 \mathrm{ml} / \mathrm{min} \times 1,73 \mathrm{~m}^{2}(\overline{\mathbf{x}} \pm 2 \mathrm{~s})$. Die von uns ebenfalls durchgeführten Bestimmungen von $b$ und $\mathrm{T}_{\mathrm{H}_{2} \mathrm{O}}^{\mathrm{C}}$ nach $\mathrm{ADH}$-Gabe können nur als ein Kriterium für die vorhandene oder fehlende Ansprechbarkeit der Niere auf ADH gewertet werden. Der bei vorhandener ADH-Ansprech-

barkeit erfolgende Anstieg von $\mathrm{T}_{\mathrm{H}_{2} \mathrm{O}}^{\mathrm{c}} \mathrm{muß}$ nicht unbedingt für das Vorliegen eines ADH-Mangels sprechen, da auch bei gesunden Probanden mit einem Anstieg dieses Meßwertes gerechnet werden muß ${ }^{3}, 8$.

\section{Ergebnisse}

1. Hypophysektomierte Patienten ohne polyurisch-polydiptisches Syndrom (Gruppe A)

Diese Patienten zeigten im 17-Std.-Durstversuch eine mit $796 \pm$ $119 \mathrm{mOsm} / \mathrm{kg}(\overline{\mathrm{x}} \pm \mathrm{s}$ ) gegenüber unserem Normalkollektiv (s. o. unter Methoden) signifikant erniedrigte $(p<0,0005)$ höchste Urinosmolalität (Tab. 1). Die ausgeschiedene Urinmenge lag zwar höher als im Normalkollektiv, differierte aber nicht signifikant. Auch zeigte sich im Mittel keine Erhöhung der Serumosmolalität. Die Ergebnisse im CarterRobbins-Test und in der osmotischen Diurese bestätigten im wesentlichen die jeweiligen Ergebnisse des Durstversuches der Patienten dieser Gruppe.

Hinzuweisen ist auf den Carter-Robbins-Test des Patienten Sch. G. (Tab. 1); er zeigt deutlich, daß die Abnahme des Urinvolumens auf die Infusion der hypertonen NaCl-Lösung allein bisweilen keine richtige Beurteilung des Testausfalls zuläßt ${ }^{\mathbf{9}},{ }^{10}$. Obwohl das Urinvolumen in diesem Fall nur um ca. $20 \%$ abnahm, stieg die Urinosmolalität ausreichend an und wurde auch durch die ADH-Gabe nur geringgradig weiter erhöht. - Be. achtenswert ist weiterhin der eindeutig normale Ausfall des Carter-RobbinsTests bei Patient F. F. (Tab. 1), obwohl im Durstversuch die höchste Urinosmolalität nur $562 \mathrm{mOsm} / \mathrm{kg}$ betrug bei einer Urinausscheidungsmenge von $1760 \mathrm{ml}$ und einem Anstieg der Serumosmolalität um $8 \mathrm{mOsm} / \mathrm{kg}$. - Andererseits lagen bei zwei Patienten (W. A., G. E.) in der osmotischen Diurese die Werte für $b$ und $T_{\mathrm{H}_{2} \mathrm{o}}^{\mathrm{c}}$ unterhalb des Normalbereiches, obwohl die höchsten Urinosmolalitäten im Durstversuch mit $689 \mathrm{bzw} .782 \mathrm{mOsm} / \mathrm{kg}$ relativ hoch lagen.

2. Patienten nach Hypophysektomie mit polyurisch-polydiptischem Syndrom (Gruppe B) und Patienten mit polyurisch-polydiptischem Syndrom anderer Genese (Gruppe C)

Bei diesen Kranken zeigte sich im Durstversuch ein im Mittel geringerer Anstieg der Urinosmolalität mit sehr viel höherer Urinausscheidungsmenge, wobei die Serumosmolalität mehr oder weniger deutlich anstieg (Tab. 1). Die Ergebnisse im Carter-Robbins-Test und in der 
Urin-Vol. max. Urin-Osm.

$\triangle$ Serum-Osm.

$=$ Urin-Volumen in $\mathrm{ml}$ pro $17 \mathrm{Std}$.

$=$ Die höchste in den einzelnen Urinportionen gemessene Osmolalität in $\mathrm{mOsm} / \mathrm{kg}$

$=$ Differenz der Serum-Osmolalität zu Beginn und am Ende des Durstversuches in mOsm/kg $(-=$ Abfall, $+=$ Anstieg)

$\Delta$ Vol. in \% nach $\mathrm{NaCl}=$ Abnahme des Urin-Volumens pro Zeiteinheit nach der Gabe einer hypertonen (2,5\%igen) NaClLösung

$\triangle$ Urin-Osm. nach $\mathrm{NaCl}=$ Anstieg der Urin-Osmolalität auf die Gabe einer hypertonen (2,5\%igen) NaCl-Lösung in mOsm/kg $\triangle$ Urin-Osm. nach ADH $=$ Anstieg der Urin-Osmolalität nach ADH-Gabe in $\mathrm{mOsm} / \mathrm{kg}$

$\mathrm{T}_{\mathrm{H}_{2} \mathrm{O}}^{\mathrm{c}}$

b
$=$ tubuläre Rückdiffusionskapazität in $\mathrm{ml}$ pro min und pro $1,73 \mathrm{~m}^{2}$ Körperoberfläche

$=$ Regressionskoeffizient für die Beziehung Urinminutenvolumen ( $V_{\text {min }}$ ) gegen die osmolale Clearance $\left(\mathrm{C}_{\text {osm }}\right)$

Weitere Einzelheiten im Text.

\begin{tabular}{|c|c|c|c|c|c|c|c|c|c|c|}
\hline \multirow[b]{2}{*}{ Patient } & \multirow{2}{*}{$\begin{array}{l}\text { Alter } \\
\text { in } \\
\text { Jahren }\end{array}$} & \multirow{2}{*}{$\begin{array}{l}\text { Zeitraum } \\
\text { seit } \\
\text { Hypophys- } \\
\text { ektomie }\end{array}$} & \multicolumn{3}{|c|}{ 17-Std.-Durstversuch } & \multicolumn{3}{|c|}{ Carter-Robbins-Test } & \multicolumn{2}{|c|}{ Osmotische Diurese } \\
\hline & & & $\begin{array}{l}\text { Urin- } \\
\text { Vol. }\end{array}$ & $\begin{array}{c}\max . \\
\text { Urin-Osm. }\end{array}$ & $\begin{array}{c}\Delta \text { Serum- } \\
\text { Osm. }\end{array}$ & $\begin{array}{l}\triangle \mathrm{Vol} . \\
\text { in \% } \\
\mathrm{n} . \mathrm{NaCl} .\end{array}$ & $\begin{array}{c}\Delta \text { Urin-Osm. } \\
\text { nach } \\
\mathrm{NaCl}\end{array}$ & $\begin{array}{c}\Delta \text { Urin-Osm. } \\
\text { nach } \\
\text { ADH }\end{array}$ & $\mathrm{T}_{\mathrm{H}_{2} \mathrm{O}}^{\mathrm{c}}$ & $\mathrm{b}$ \\
\hline
\end{tabular}

A. Patienten nach Hypophysektomie ohne polyurisch-polydiptisches Syndrom

\begin{tabular}{|c|c|c|c|c|c|c|c|c|c|c|}
\hline đ Ro. A. & 13 & 2 Wo. & 780 & 608 & -4 & -- & - & $\ldots$ & - & - \\
\hline o Ro. A. & 13 & 9 Wo. & 508 & 1058 & +5 & $\cdots-$ & - & $\cdots \cdots$ & - & - \\
\hline o Z. X. & 62 & 9 Wo. & 526 & 779 & --4 & - & $\ldots$ & - & - & - \\
\hline$d$ G. P. & 60 & 2 Wo. & 1045 & 681 & -2 & - & $\cdots$ & - & - & - \\
\hline 오. R. & 17 & 3 Mon. & 415 & 854 & +1 & $\ldots$ & - & - & - & - \\
\hline o Ru. A. & 61 & $4 \mathrm{~J}$ & 306 & 1000 & 0 & $\ldots$ & $\longrightarrow$ & $\longrightarrow$ & - & - \\
\hline o Sch. J. & 37 & 3 Mon. & 662 & 855 & +3 & - & -- & - & - & 一 \\
\hline 오. F. & 65 & $12 \mathrm{Tg}$ & 540 & 750 & -4 & -- & - & - & - & - \\
\hline đW. W. & 54 & 3 Wo. & 1190 & 678 & +6 & $-\cdots$ & - & 一 & - & - \\
\hline$\Leftrightarrow \mathrm{A} . \mathrm{H}$ & 62 & $3 \mathrm{~J}$ & 710 & 783 & -8 & - & $\ldots$ & - & - & - \\
\hline đ K. E. & 63 & $14 \mathrm{~J}$ & 2035 & 720 & -1 & - & - & - & - & - \\
\hline o R.J. & 48 & 31/2 Mon. & 592 & 899 & +4 & 80,4 & +447 & 一 & - & - \\
\hline क F. F. & 40 & 2 Wo. & 1763 & 562 & +8 & $-72,5$ & +434 & +40 & - & - \\
\hline
\end{tabular}




\begin{tabular}{|c|c|c|c|c|c|c|c|c|c|c|}
\hline ठ E. K. & 36 & $4 \mathrm{~J}$ & 718 & 988 & +4 & $--79,4$ & +543 & +50 & - & - \\
\hline đSch. G. & 45 & $3 \mathrm{~J}$ & 642 & 788 & +3 & $\cdots 19,7$ & +367 & +42 & -- & - \\
\hline ๙ Sch. M. & 33 & 20 Mon. & 1186 & 800 & -3 & -- & - & $\ldots-$ & 4,82 & 1,08 \\
\hline Q E. K. & 50 & 12 Mon. & 560 & 705 & +1 & $\longrightarrow$ & $\ldots$ & - & 4,41 & 1,10 \\
\hline J E. F. & 54 & $3 \mathrm{~J}$. & 720 & 807 & -4 & $\ldots$ & -- & $\cdots$ & 2,51 & 0,98 \\
\hline$\approx \mathrm{W}, \mathrm{A}$. & 28 & 10 Wo. & 1387 & 689 & $-\cdots-1$ & $\ldots$. & - & - & 0,43 & 0,86 \\
\hline б G. E. & 66 & $4 \boldsymbol{J}$ & 293 & 782 & +6 & - & -- & $\longrightarrow$ & $-0,5$ & 0,84 \\
\hline ð Schn. M. & 47 & $4 \mathrm{~J}$ & 440 & 915 & +1 & $\ldots$ & - & - & 4,28 & 1,06 \\
\hline$\approx \mathrm{L} . \mathrm{G}$ & 31 & 16 Mon. & 760 & 772 & +3 & - & - & $\cdots$ & 5,07 & 0,93 \\
\hline$\partial A . W$. & 55 & $21 / 2 \mathrm{~J}$ & 763 & 850 & -7 & - & - & $-\cdots$ & 2,54 & 0,89 \\
\hline
\end{tabular}

B. Patienten nach Hypophysektomie mit polyurisch-polydiptischem Syndrom

\begin{tabular}{|c|c|c|c|c|c|c|c|c|c|c|}
\hline$\delta \mathrm{S} . \mathrm{L}$. & 27 & $3 \mathbf{J}$ & 5160 & 159 & +12 & - & - & - & & - \\
\hline ๙ S. H. & 19 & $7 \mathrm{~J}$ & 697 & 684 & +1 & - & - & - & - & - \\
\hline Scho. G. & 22 & $14 \mathrm{~J}$. & 4018 & 130 & $\begin{array}{l}+8 \\
+\quad 8\end{array}$ & + 78,9 & +10 & +384 & - & - \\
\hline o R. F. & 55 & $4 \mathrm{~J}$ & 2120 & 401 & +8 & +152 & +62 & +212 & - & - \\
\hline ð St. K. & 21 & $1 \mathrm{~J}$ & 2660 & 140 & + & +100 & -74 & +302 & - & - \\
\hline o K. H. & 31 & $1 \mathrm{~J}$ & 1683 & 366 & +7 & $\begin{array}{r}+\quad 39,5 \\
\end{array}$ & -1 & +499 & - & - \\
\hline o H. J. & 55 & $3 \mathrm{~J}$. & 2774 & 243 & +6 & - & - & - & $-6,1$ & 0,7 \\
\hline
\end{tabular}

C. Patienten mit polyurisch-polydiptischem Syndrom (keine Hypophysektomie)

\begin{tabular}{|c|c|c|c|c|c|c|c|c|c|c|}
\hline$\approx \mathrm{K} . \mathrm{K}$ & 28 & Tumor? & 780 & 553 & $t$ & t. 56,1 & + & +506 & - & - \\
\hline \&W. K. & 33 & Idiopathisch & 3410 & 248 & + & +65 & 50 & +373 & - & - \\
\hline ¿ M. W. & 28 & $\begin{array}{l}\text { Psychogene } \\
\text { Polydipsie }\end{array}$ & 1204 & 729 & +4 & $-81,8$ & +336 & +111 & - & - \\
\hline o Sm. G. & 23 & $\begin{array}{l}\text { Diabetische } \\
\text { Nephropathie }\end{array}$ & 3450 & 303 & +24 & 9,4 & $t$ & + & - 5,4 & 0,77 \\
\hline ㅇ. B. M. & 55 & Tumor & 2432 & 288 & +17 & - & - & - & $-12,1$ & 0,45 \\
\hline 果 H. L. & 19 & Idiopathiseh & 1850 & 312 & +20 & - & $\ldots$ & - & $-\quad 5,87$ & 0,74 \\
\hline
\end{tabular}


osmotischen Diurese bestätigten mit einer Ausnahme den Ausfall der Durstversuche.

Der Patient K. K. zeigte im Carter-Robbins-Test, obwohl die Urinosmolalität im Durstversuch auf $553 \mathrm{mOsm} / \mathrm{kg}$ bei einer Urinausscheidungsmenge von nur $780 \mathrm{ml}$ angestiegen war, praktisch überhaupt keinen Anstieg der Urinosmolalität auf die hypertone NaCl-Lösung mit einem starken Anstieg der Urinosmolalität auf die ADH-Gabe (Tab. 1). Es mußte also eine deutliche Einschränkung der Konzentrierfähigkeit diagnostiziert werden.

Sonderfälle: Patient Sm. G. (Tab. 1) zeigte in allen drei Testen eindeutig pathologische Befunde. Da im Carter-Robbins-Test auch auf die Gabe von $\mathrm{ADH}$ ein nur ungenügender Anstieg der Urinosmolalität zu erzielen war, mußte also bei ,diabetischer Nephropathie" (überwiegend eine Pyelonephritis mit Hydronephrose und neurogener Blasenentleerungsstörung) ein erworbener renaler Diabetes insipidus angenommen werden. Eine endogene osmotische Diurese (hyperglykämisch-glukosurisch bzw. azotämisch) konnte ausgeschlossen werden, da die Blutzuckerspiegel zum Zeitpunkt der Testdurchführung stets um $200 \mathrm{mg} \%$ bzw. niedriger lagen und eine Azotämie nicht vorlag (Inulin- bzw. PAH-Clearance an der untersten Normgrenze bzw. geringgradig erniedrigt). - Zwei Patienten aus diesen beiden Gruppen zeigten eine nahezu normale Konzentrierfähigkeit. So konnte Patient S. H. (Gruppe B, Tab. 1), obwohl er klinisch ein polyurisch-polydiptisches Syndrom mit Ausseheidungsmengen von mehr als 2 Litern bot, seinen Urin bis zu $685 \mathrm{mOsm} / \mathrm{kg}$ konzentrieren und schied während der $17 \mathrm{Std}$. nur ca. $700 \mathrm{ml}$ aus. Es lag hier also höchstens ein inkompletter Diabetes insipidus vor, möglicherweise vorgetäuscht durch eine psychogene Komponente. Es wäre nämlich denkbar, daß dieser Patient, bei dem die Hypophysektomie um Jahre zurücklag, zunächst postoperativ eine polyurisch-polydiptische Phase durchgemacht und sich zwischenzeitlich an die größeren Trinkmengen „gewöhnt" hat, obwohl zwischenzeitlich - wie das Ergebnis des Durstversuches zeigt - eine ausreichende ADH-Sekretion wieder möglich war (primäre Polydipsie? ${ }^{11}$ ). - Das polyurisch-polydiptische Syndrom von Patient M. W. (Gruppe C, Tab. 1) mußte dagegen als psychogene Polydipsie gedeutet werden, da nach den Ergebnissen im Durstversuch und im Carter-Robbins-Test kein Anhalt für eine eindeutige Konzentrierschwäche zu finden und keine hypothalamisch-hypophysäre Erkrankung vorausgegangen war. Daß die Harnkonzentrationswerte gegenüber denen von Normalpersonen etwas erniedrigt sind, läßt sich durch die Einschränkung der Konzentrierfähigkeit der Niere infolge andauernder Ủberwässerung erklären ${ }^{6},{ }^{12}$.

Den Vergleich der Ergebnisse des aufwendigeren und den Patienten sehr viel mehr belastenden Tests (Carter-Robbins-Test bzw. osmotische Diurese) mit dem Ergebnis des beim jeweiligen Patienten ebenfalls durchgeführten 17-Std.-Durstversuches zeigt Abb. 1. Der Ausfall des Durstversuches korreliert gut mit dem am selben Patienten ermittelten Ergebnis des Carter-Robbins-Tests $(r=0,91, \mathrm{p}<0,001)$ bzw. mit dem Ergebnis der osmotischen Diurese $(r=0,92, p<0,001)$, obwohl in diesem Vergleich Patienten mit pathologischen und nicht pathologischen Befunden unausgewählt einbezogen sind. 


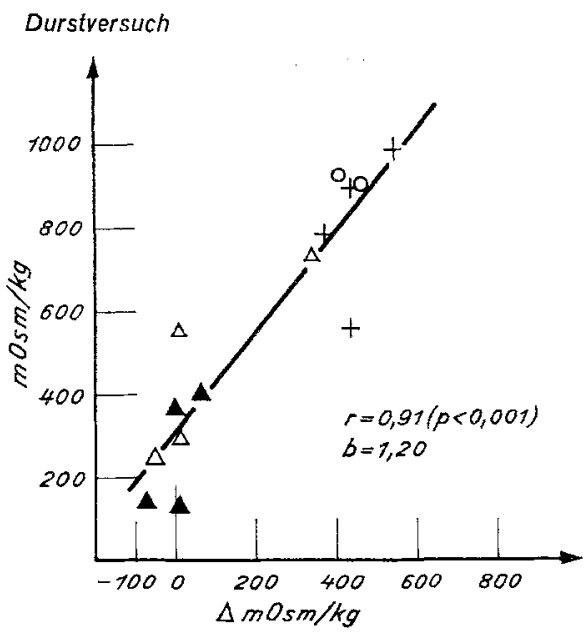

Carter-Robbins-Test

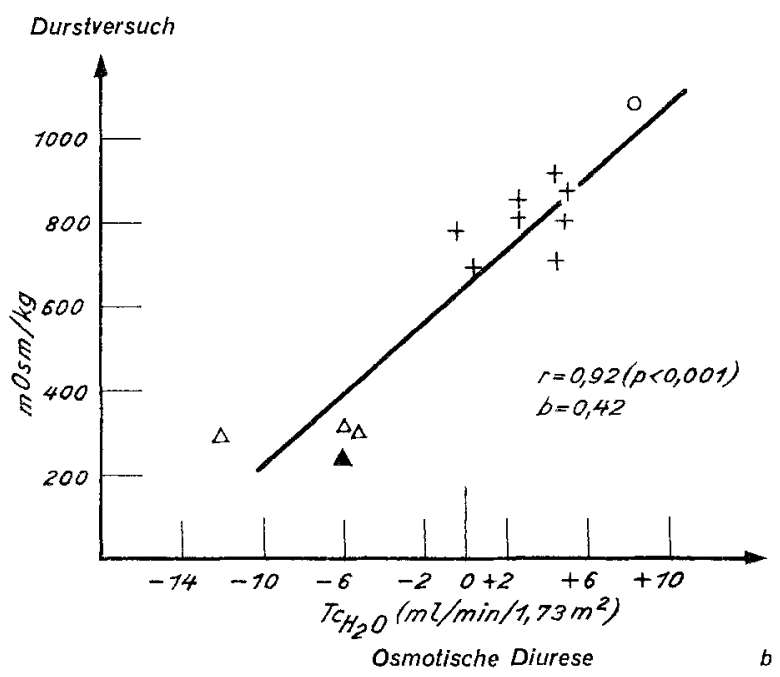

Abb. 1. Korrelation zwischen den Ergebnissen des 17-Std.-Durstversuches (wichtigstes Kriterium: höchste Urinosmolalität) und des am selben Patienten durohgeführten Carter-Robbins-Testes (wichtigstes Kriterium: Anstieg der Urinosmolalität auf die Infusion der hypertonen NaCl-Lösung) bzw. der osmotischen Diurese (wichtigstes Kriterium : die tubuläre Rückdiffusionskapazität $\mathrm{T}_{\mathrm{H}_{2} \mathrm{O}}^{\mathrm{c}}$ ). Die Korrelationen sind in beiden Fällen hochsignifikant von Null verschieden. $\mathrm{O}=$ Normalpersonen. $+=$ Hypophysektomierte Pat. ohne polyurisch-polydiptisches Syndrom. $\mathbf{A}=$ Hypophysektomierte Pat. mit polyurisch-polydiptischem Syndrom. $\Delta=$ Pat. mit polyurischpolydiptischem Syndrom anderer Genese 


\section{Diskussion}

Die diagnostische Aussagekraft eines exakt durchgeführten Durstversuches ist nach dieser Untersuchung beim Diabetes insipidus groß. $\mathrm{Zu}$ fordern sind: 1. Die Bestimmung der Osmolalität in Serum und Urin, und nicht die Messung des ungenaueren spezifischen Gewichtes 2, 3, 4, das keine ausreichend genaue Differenzierung des Schweregrades eines Diabetes insipidus zuläßt. 2. Eine exakte Gewichtskontrolle der Patienten vor Beginn und nach Beendigung des Durstversuches, da eine Diskrepanz zwischen Urinausscheidungsmenge und Gewichtsabnahme eine Flüssigkeitszufuhr während der Durstperiode aufdeckt. Auch ist bei eindeutig pathologischem Ausfall des Durstversuches (Urinosmolalität < $400 \mathrm{mOsm} / \mathrm{kg}$ ) ein Anstieg der Serumosmolalität ( $\geqslant 6 \mathrm{mOsm} / \mathrm{kg}$ ) obligatorisch. Ist die Differentialdiagnose zwischen einem ADH-Mangel und einer mangelhaften Ansprechbarkeit der Niere auf ADH nicht schon „ex juvantibus" durch einen Therapieversuch mit ADH-Präparaten geklärt, muß zusätzlich zum Durstversuch die ADH-Wirksamkeit überprüft werden (bei uns nach Carter-Robbins-Test bzw. osmotischer Diurese). Abgesehen davon kann ein Durstversuch im Einzelfall trotz der guten Korrelation zwischen dem am gleichen Patienten durchführten Durstversuch und dem Carter-Robbins-Test bzw. der osmotischen Diurese ein zweifelhaftes Ergebnis bringen. Unter 23 Patienten, bei denen außer einem Durstversuch noch einer der beiden anderen Teste durchgeführt wurde, zeigten 2 Patienten (F. F., K. K.) mit höchsten Urinosmolalitäten um $550 \mathrm{mOsm} / \mathrm{kg}$ ein nicht eindeutiges Ergebnis. Der Patient F. F. zeigte im 24 Stunden nach dem Durstversuch durchgeführten Carter-Robbins-Test ein normales Verhalten. Hierbei lag der Anstieg der Serumosmolalität auf die hypertone NaCl-Infusion mit $5 \mathrm{mOsm} / \mathrm{kg}$ nicht höher als im Durstversuch (Tab. 1). Es bleibt aber die Möglichkeit, daß nicht der Absolutwert, sondern die Steilheit des Anstieges der Serumosmolalität im Carter-Robbins-Test größer als im Durstversuch ist und dadurch ein - in diesem Fall operativ - geschädigtes hypothalamisch-hypophysäres System besser anspricht ${ }^{1}$. Extrarenale Faktoren, die die Hyposthenurie im Durstversuch erklären könnten, z. B. latente Ödeme, waren nicht zu ermitteln. Im Gegensatz dazu steht das Ergebnis des Carter-Robbins-Tests bei Patient K. K., der im Durstversuch immerhin ebenfalls eine Urinosmolalität um $550 \mathrm{mOsm} / \mathrm{kg}$ erreicht hatte. Der fehlende Anstieg der Urinosmolalität auf die 2,5\% ige NaCl-Lösung mit dem deutlichen Anstieg auf die anschließende ADH-Gabe sprach dafür, daß in diesem Fall auch durch stärkere Stimuli als das Dursten keine wesentliche ADH-Mehrausschüttung erfolgte. Daß außerdem zwoi weitere Patienten (W. A., G. E.) mit einer höchsten Urinosmolalität im Durstversuch um $700 \mathrm{~m} 0 \mathrm{sm} / \mathrm{kg}$ 
unter der größeren Belastung in der osmotischen Diurese bereits unter dem Normalbereich liegende Werte aufwiesen, fällt dagegen nicht so sehr ins Gewicht, da bei einer bedeutsamen Störung der Konzentrierfähigkeit die osmotische Diurese weit pathologischere Ergebnisse zeigt (Tab. 1).

Unser diagnostisches Vorgehen ist daher folgendes: Bei polyurischpolydiptischem Syndrom bzw. zum Ausschluß einer Konzentrationsstörung, z. B. nach Hypophyselktomie, führen wir zunächst einen 17 Std.-Durstversuch durch. Steigt die höchste Urinosmolalität nicht über $500 \mathrm{mOsm} / \mathrm{kg}$ an, liegt auch abgesehen von der klinischen Manifestation (Polyurie) eine eindeutige Einschränkung der Konzentrierfähigkeit vor. Bei Werten ab $750 \mathrm{mOsm} / \mathrm{kg}$ liegt dagegen keine erhebliche Konzentrationsstörung vor. Lediglich der Bereich zwischen 500 und $750 \mathrm{mOsm} / \mathrm{kg}$ erfordert zur endgültigen, exakten Diagnose ein weiteres Vorgehen. Nur dann wird - unter Berücksichtigung der Zumutbarkeit der weiteren Diagnostik - einer der beiden anderen Teste zusätzlich angewandt. Wegen der etwas einfacheren Durchführbarkeit ziehen wir den CarterRobbins-Test vor, obwohl die Untersuchung mit osmotischer Diurese eine genauere Differenzierung und Quantifizierung des Konzentrierdefektes erlaubt, die aber für die Praxis entbehrlich ist.

DaB die Untersuchungen an hypophysektomierten Patienten auch in der Gruppe ohne polyurisch-polydiptisches Syndrom ein gegenüber Normalpersonen signifikant geringeres Konzentrationsvermögen ergaben - wie auch von anderen Autoren nach Hypophysektomie beschrieben ${ }^{1,13}$-, muß im Sinne einer geringeren ,ADH-Reserve" gedeutet werden, die sich klinisch jedoch noch nicht bemerkbar machte. Ob die Verschiedenheit der Operationsverfahren und der Tumorausdehnung die Konzentrierfähigkeit unterschiedlich stark beeinflussen, kann anhand dieser Untersuchungen in Anbetracht der für eine Aufschlüsselung nach den verschiedenen Operationsmethoden zu kleinen Fallzahl und des zu unterschiedlichen, nach der Hypophysektomie verstrichenen Zeitraumes (Tab. 1) nicht gesagt werden. Die Patienten, bei denen relativ kurzzeitig nach Hypophysektomie ( 2 bis 10 Wochen) ein Durstversuch durchgeführt werden konnte, hatten meist eine deutlichere Einschränkung der Konzentrierfähigkeit, auch wenn kein polyurisch-polydiptisches Syndrom vorlag. Zur genauen Beantwortung der Frage nach dem Einfluß der seit Hypophysektomie verstrichenen Zeit auf die Konzentrationsleistung, müßten wiederholte Untersuchungen an denselben Patienten zu verschiedenen Zeitpunkten nach Hypophyselztomie durchgeführt werden. 


\section{Literatur}

1. Buchborn, E., und K. Irmscher, Diabetes insipidus bei Tumoren der Hypophyse. Symp. Dtsch. Ges. Endokrin. 15 (1969), 256-262.

2. Hirsch, F., Zur klinischen Diagnostik des Diabetes insipidus. Dissertation 1971, München.

3. Buchborn, E., Störungen der Harnkonzentrierung. In: Handbuch der Inneren Medizin, 5. Aufl., Nierenkrankheiten, Bd. VIII/1, S. 491-673. Hrsg. H. Schwiegk. Berlin-Heidelberg-New York: Springer. 1968.

4. Isaacson, L. C., Urinary osmolality and specific gravity. Lancet (1959) I, $72-73$.

5. Carter, A. C., and J. Robbins, The use of hypertonic saline infusions in the differential diagnosis of diabetes insipidus and psychogenic polydipsia. J. clin. Endocr. 7 (1947), $753-766$.

6. Price, J. D. F., and R. W. Lauener, Serum and urine osmolalities in the differential diagnosis of polyuric states. J. clin. Endocr. 26 (1966), $143-148$.

7. Irmscher, K., Zur Klinik des Diabetes insipidus. Habilitationsschrift 1967, Düsseldorf.

8. Zak, G. A., C. Brun, and H. W. Smith, The mechanism of formation of osmotically concentrated urine during the antidiuretic state. J. clin. Invest. 33 (1954), 1064-1074.

9. Moses, A. M., and D. H. P. Streeten, Differentiation of polyuric states by measurement of response to changes in plasma osmolality induced by hypertonic saline infusions. Amer. J. Med. 42 (1967), 368-377.

10. Barlow, E. D., and H. E. de Wardener, Compulsive water drinking. Quart. J. Med. 28 (1959), 235-258.

11. Pickardt, C. R., und R. Fahlbusch, Chronische Hyperosmolalität, Adipsie und ,sekundäre" Hypophysenvorderlappeninsuffizienz bei Hypothalamus-Schädigung. Internist 13 (1972), 45-51.

12. Epstein, F. H., C. R. Kleeman, and A. Hendricx, The influence of bodily hydration on the renal concentrating process. J. clin. Invest. 36 (1957), 629-634.

13. Lipsett, M. B., and O. H. Pearson, Further studies of diabetes insipidus following hypophysectomy in man. J. Lab. clin. Med. 49 (1947), 190-199.

Anschrift der Verfasser: Dr. med. O. A. Müller, Dr. med. S. Ascher, Dr. med. F. Hirsch, Dr. med. F. Kluge, Prof. Dr. med. H. C. Moll, Prof. Dr. med. P. C. Scriba, II. Medizinische Universitätsklinik, Ziemssenstraße 1, D-8 München 2, Deutschland, Dr. med. R. Fahlbusch, Neurochirurgische Universitätsklinik, Beethovenplatz 2-3, D-8 München 2, Deutschland. 